\title{
Elevated interleukin-6 expression levels are associated with intervertebral disc degeneration
}

\author{
XIAO DENG, FENG ZHAO, BAOLIN KANG and XIN ZHANG \\ Department of Orthopedics, Shaanxi Sengong Hospital, Xi'an, Shaanxi 710300, P.R. China
}

Received December 22, 2014; Accepted September 15, 2015

DOI: $10.3892 /$ etm.2016.3079

\begin{abstract}
The present study aimed to investigate whether serum interleukin-6 (IL-6) expression levels were associated with the onset and progression of intervertebral disc degeneration (IDD). A comprehensive meta-analysis of the scientific literature from numerous electronic databases was performed, in order to obtain published studies associated with the topic of interest. Relevant case-control studies that had previously assessed a correlation between IL-6 expression levels and IDD were identified using predetermined inclusion and exclusion criteria. The STATA version 12.0 software was used for statistical analysis of the extracted data. A total of 112 studies were initially retrieved, with eight studies meeting the inclusion criteria. These contained a total of 392 subjects, of which 263 were patients with IDD and 129 were healthy controls. A meta-analysis of the eight studies demonstrated that serum IL-6 protein expression levels may be associated with IDD, and this was irrespective of IDD subtype (bulging, protrusion, or sequestration). Notably, serum expression levels of the IL-6 protein were upregulated in intervertebral disc (IVD) protrusion tissue, as compared with normal IVD tissue; thus suggesting that IL-6 may have an important role in the pathophysiological process of IDD.
\end{abstract}

\section{Introduction}

Intervertebral disc degeneration (IDD), which is considered to be a global health threat, is a gateway for other disc-related diseases and is associated with significant healthcare costs $(1,2)$. IDD precedes, or is associated with, numerous clinical conditions, including lower back or limb pain, spinal stenosis, and disc herniation (3). Clinically, IDD is characterized by a loss of intervertebral disc (IVD) height and structural failure, due to proteolytic degradation

Correspondence to: Dr Xiao Deng, Department of Orthopedics, Shaanxi Sengong Hospital, 9 Huazhan Road, Xi'an, Shaanxi 710300, P.R. China

E-mail: dengxiao607@163.com

Key words: interleukin-6, intervertebral disc, intervertebral disc degeneration, meta-analysis of the extracellular matrix (ECM) and infiltration of blood vessels and nerve fibers $(2,4)$. IDD is generally accepted as an inescapable consequence of the aging process; it affects $90-100 \%$ of individuals aged $>63$ years, and is the predominant cause of $\sim 40 \%$ of cases of lower back pain $(5,6)$. As a multifactorial disease, IDD may be caused by genetic or environmental factors, among which mechanical stress to the spine is the primary factor, although age, heavy lifting, smoking, and biochemical influences may also contribute to the risk of IDD $(7,8)$. Currently, there is no cure for IDD, and it is instead managed via surgical procedures, including vertebral fusion and disc excision, which may relieve the pain in the short term but alter the biomechanics of the spine in the long term, resulting in degeneration of the surrounding tissues and adjacent discs $(9,10)$. Miyagi et al (11) detected elevated levels of interleukin-6 (IL-6) in the sera of patients with IDD; therefore, the present meta-analysis aimed to investigate the association of IL-6 with IDD, in order to hasten the development of early therapeutic intervention strategies.

IL-6 is a $26-\mathrm{kDa}$ protein, which was initially described as a B-cell activating factor secreted by T-cells, and can be mapped to chromosome 7p15-p21 (12). IL-6 is a well-established pro-inflammatory cytokine that stimulate the growth and proliferation of numerous immune cell types during host immune defense responses (13). Furthermore, it is considered to be a key regulator of human chronic inflammatory diseases (14). IL-6 is able to initiate various effects on the nervous system, vascular tissue, immune response and stress response, by modulating gene expression and cell survival, proliferation, and differentiation (15). Numerous studies have associated elevated serum levels of IL- 6 with cardiovascular diseases, type 2 diabetes, rheumatoid arthritis, multiple sclerosis, Crohn's disease, and lymphatic, renal, bladder and colorectal cancers $(13,16)$. In addition, IL-6 has been associated with IDD pathogenesis (17-19); however, Liu et al (20) was unable to detect an association between IL-6 and disc-associated disorders. Within a meta-analysis framework, the present study investigated the correlation between serum IL-6 protein expression levels and IDD pathology, with the intention of developing early intervention strategies.

\section{Materials and methods}

Search strategy. The present study was conducted on the basis of the guidelines of the Preferred Reporting Items for 
Systematic Reviews and Meta-analysis (PRISMA guidelines; http://www.prisma-statement.org/). A comprehensive and systematic literature search of numerous electronic databases, including PubMed (http://www.ncbi.nlm.nih. gov/pubmed/), EBSCO (http://search.ebscohost.com/), Ovid (http://gateway.ovid.com/), Medline (http://www.medline. com/), Springerlink (http://link.springer.com/), Wiley Online Library (http://onlinelibrary.wiley.com/), Web of Science (http://wok.mimas.ac.uk/), the Chinese Biomedical Database (http://www.sinomed.ac.cn/), the Chinese Journal Full-Text Database (part of the China National Knowledge Infrastructure; http://www.cnki.net/), the Wanfang Database (http://www.wanfangdata.com.cn/) and the VIP Database (http://www.cqvip.com/), was performed. In addition, the reference lists from numerous original and review articles were manually searched. A combination of Medical Subject Headings, Medline medical index terms and free words, were used to retrieve studies broadly associated with the topic of interest. The search criteria were as follows: 'Intervertebral disc degeneration'; 'intervertebral disk degeneration'; 'disk degeneration'; 'disk degradation'; 'disc degeneration'; ‘disc degradation'; 'degeneration, disc'; 'degenerative intervertebral disc'; 'degenerative intervertebral disks'; 'lumbar disc herniation'; 'disc herniation'; 'disk herniation'; ‘cervical disc herniation'; 'lumbar intervertebral disc herniation' or 'lumbar intervertebral disk herniation'; and 'interleukin-6'; 'plasmacytoma growth factor'; 'B-cell differentiation factor-2'; 'B-cell stimulatory factor 2'; 'BSF-2'; 'hepatocyte-stimulating factor'; 'hybridoma growth factor'; 'IFN-beta 2'; 'IL-6'; 'IL 6'; 'MGI-2'; 'myeloid differentiation-inducing protein'; 'B cell stimulatory factor-2'; or 'B-cell differentiation factor'.

Inclusion and exclusion criteria. The published studies were included in the meta-analysis if they met the following inclusion criteria: i) It was a case-control study, which had investigated the association between the expression levels of IL-6 and IDD; ii) the study had included patients with IDD and healthy controls; iii) complete data, including sample size, age, ethnicity, gender, pathological types and the IL-6 protein expression levels, were available; and iv) only the study with the largest sample size or the most recent study was selected when the retrieved studies were published by the same authors using identical case materials. The exclusion criteria were as follows: i) The study was not relevant to the study topic; ii) it was not a case-control study; iii) the data was incomplete; iv) the study was from a non-English or non-Chinese publication; or v) the study was a duplicate.

Data extraction and quality assessment. Two independent investigators used a standard reporting form to extract data from each study. The following information was collated: Initials of the first author, year of submission, the country of submission, language, ethnicity, study design, disease, protein detection method, total number of included cases, age, expression levels of IL-6, and the type of IDD. Disagreements were resolved by reaching a consensus following a discussion with numerous investigators during data extraction. The quality of the selected studies were estimated independently by two investigators based on the critical appraisal skills program
(CASP) score criteria (http://www.casp-uk.net/), which are as follows: i) Unambiguous study issue (CASP01); ii) an appropriate method was used to answer study issue (CASP02); iii) acceptable case selection (CASP03); iv) acceptable selection of controls (CASP04); v) accurate measure of exposure factors for minimizing bias (CASP05); vi) other confounding factors were controlled (CASP06); vii) complete study results were available (CASP07); viii) precise study results (CASP08); ix) credible study results (CASP09); x) suitable study results for the local population (CASP10); and xi) consensus between the study results and other evidence (CASP11).

Statistical analysis. The correlations between the IL-6 protein expression levels and IDD were estimated using the standardized mean difference (SMD) and the corresponding 95\% confidence interval (95\% CI). The pooled SMDs were determined using the Z-test. The heterogeneity among the various studies was quantified using the Cochran's Q-statistic and the $\mathrm{I}_{2}$ index $(21,22)$. A random-effects model was used in the case of significant heterogeneity $\left(\mathrm{P}<0.05\right.$ or $\left.\mathrm{I}_{2}>50 \%\right)$, and a fixed-effects model was used in the case of non-significant heterogeneity (23). The potential sources of heterogeneity were assessed via single-factor or multi-factor meta-regression analyses, and the Monte Carlo simulation was used for multiple calibration tests. Subsequently, sensitivity analyses were performed, in order to evaluate whether removing a single study influenced the results. Funnel plots alongside the Egger's linear regression test were used to detect the publication bias of the selected studies. All statistical analyses were performed using the STATA version 12.0 software (StataCorp LP, College Station, TX, USA).

\section{Results}

Baseline characteristics of included studies. Fig. 1 demonstrates the screening process of the included studies. A total of 112 publications were retrieved through electronic database and manual searches, of which 104 were excluded for the following reasons: 14 studies were duplicates; two were letters or review articles; 11 involved non-human subjects; 44 were not associated with the topic of interest; 11 had insufficient data; and 22 possessed data of a low relevancy. Eight eligible case-control studies (24-31) were pooled for the present meta-analysis, which analyzed the serum IL-6 protein expression levels in 263 IDD cases (with bulging, protrusion and sequestered IDD subtypes), as compared with 129 controls. The eight studies were published between 2002 and 2014, and included five studies involving Chinese subjects and three studies involving Caucasians (one of each from Turkey, Italy and Brazil). All of the included studies determined serum IL-6 protein expression levels using ELISA. The baseline characteristics and CASP quality assessments of the selected studies are presented in Table I and Fig. 2, respectively.

Patients with IDD vs. normal individuals. Tests indicated the existence of heterogeneity in the IL- 6 protein expression levels of patients with IDD; therefore, a random-effects model was used $\left(\mathrm{P}<0.001 ; \mathrm{I}_{2}=95.9 \%\right)$. The IL-6 protein expression levels 
Table I. Baseline characteristics of the included studies.

\begin{tabular}{|c|c|c|c|c|c|c|c|c|c|}
\hline \multirow[b]{2}{*}{$\begin{array}{l}\text { First author } \\
\text { (reference) }\end{array}$} & \multirow[b]{2}{*}{ Year } & \multirow[b]{2}{*}{ Ethnicity } & \multirow[b]{2}{*}{ Total } & \multicolumn{3}{|c|}{ Case } & \multicolumn{3}{|c|}{ Control } \\
\hline & & & & $\mathrm{N}$ & $\begin{array}{c}\text { Age } \\
\text { (years) }\end{array}$ & $\begin{array}{c}\text { Gender } \\
(\mathrm{M} / \mathrm{F})\end{array}$ & $\mathrm{N}$ & $\begin{array}{c}\text { Age } \\
\text { (years) }\end{array}$ & $\begin{array}{c}\text { Gender } \\
(\mathrm{M} / \mathrm{F})\end{array}$ \\
\hline Ouyang (24) & 2014 & Asian & 70 & 50 & $43.9(22-58)$ & $31 / 19$ & 20 & $41.4(27-65)$ & $15 / 5$ \\
\hline Ding (25) & 2014 & Asian & 50 & 30 & $41.32 \pm 7.24$ & $19 / 11$ & 20 & $42.35 \pm 6.49$ & $12 / 8$ \\
\hline Li (26) & 2010 & Asian & 80 & 60 & $42.7 \pm 11.4$ & $38 / 22$ & 20 & $41.5 \pm 9.3$ & $13 / 7$ \\
\hline Kraychete (27) & 2010 & Mixed & 33 & 23 & $42.8 \pm 7.0$ & $12 / 11$ & 10 & $39.5 \pm 4.5$ & $6 / 4$ \\
\hline Song (28) & 2009 & Asian & 50 & 30 & $46.1(34-57)$ & $22 / 8$ & 20 & $42.5(29-60)$ & $15 / 5$ \\
\hline Demircan (29) & 2007 & Caucasian & 32 & 12 & $42-63$ & $20 / 12$ & 20 & - & - \\
\hline Dong (30) & 2004 & Asian & 40 & 28 & $45.4(35-53)$ & $21 / 7$ & 12 & - & $9 / 3$ \\
\hline Specchia (31) & 2002 & Caucasian & 37 & 30 & $40.7 \pm 6.3$ & $19 / 11$ & 7 & $42.2 \pm 5.9$ & - \\
\hline
\end{tabular}

Age appears as the mean (range) or as the mean \pm standard deviation. The method used in all studies was ELISA. N, number; M, male; F, female.

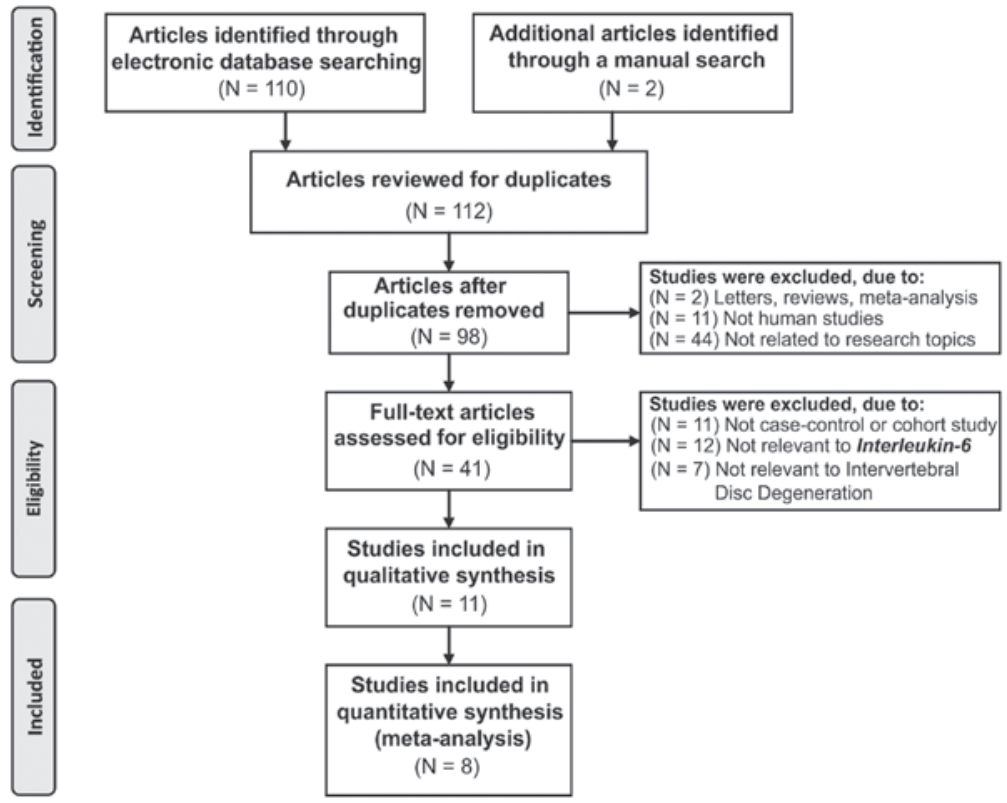

Figure 1.A flow chart of the screening process for included studies, and the exclusion criteria,based on the PRISMA guidelines(http://www.prisma-statement.org/). A total of eight case-control studies were included in the present meta-analysis.

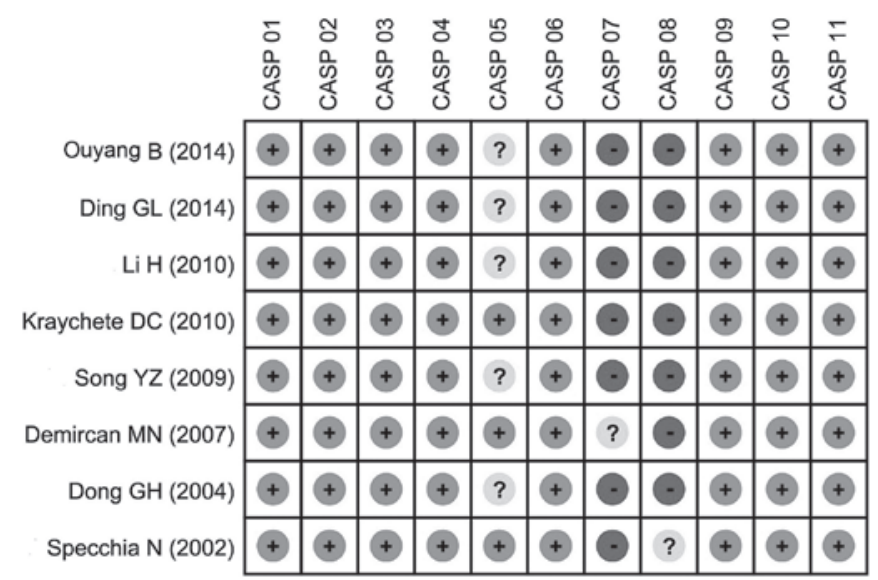

Figure 2. Methodological quality assessments for each of the included case-control studies using the Critical Appraisal Skill Program (CASP) score. The studies appearing in the figure correspond to references 24-31 (from top to bottom). +, low risk; ?, unidentified risk; -, high risk. markedly correlated with IDD (SMD=3.55; 95\% CI=1.77-5.32; $\mathrm{P}<0.001$; Fig. 3A).

Patients with IDD subtypes vs. normal individuals. A fixed-effects model was used due to the lack of heterogeneity in the IL-6 protein expression levels in patients with the various IDD subtypes, including bulging, protrusion and sequestered IDD (bulging: $\mathrm{P}=0.757, \mathrm{I}_{2}=0.0 \%$; protrusion: $\mathrm{P}=0.285, \mathrm{I}_{2}=12.4 \%$; sequestered: $\mathrm{P}=0.230, \mathrm{I}_{2}=30.6 \%$ ). All of the IDD subtypes exhibited upregulation of the IL-6 protein, as compared with the controls, which gradually increased with the severity of IDD (bulging: $\mathrm{SMD}=2.48,95 \% \mathrm{CI}=1.78-3.18$, $\mathrm{P}<0.01$; protrusion: $\mathrm{SMD}=3.33,95 \% \mathrm{CI}=2.64-4.02, \mathrm{P}<0.01$; sequestered: $\mathrm{SMD}=7.51,95 \%$ CI=6.11-8.91, P<0.01; Fig. 3B-D).

Sensitivity analysis and publication bias. The sensitivity analyses demonstrated that no single publication exerted 
A

IL-6

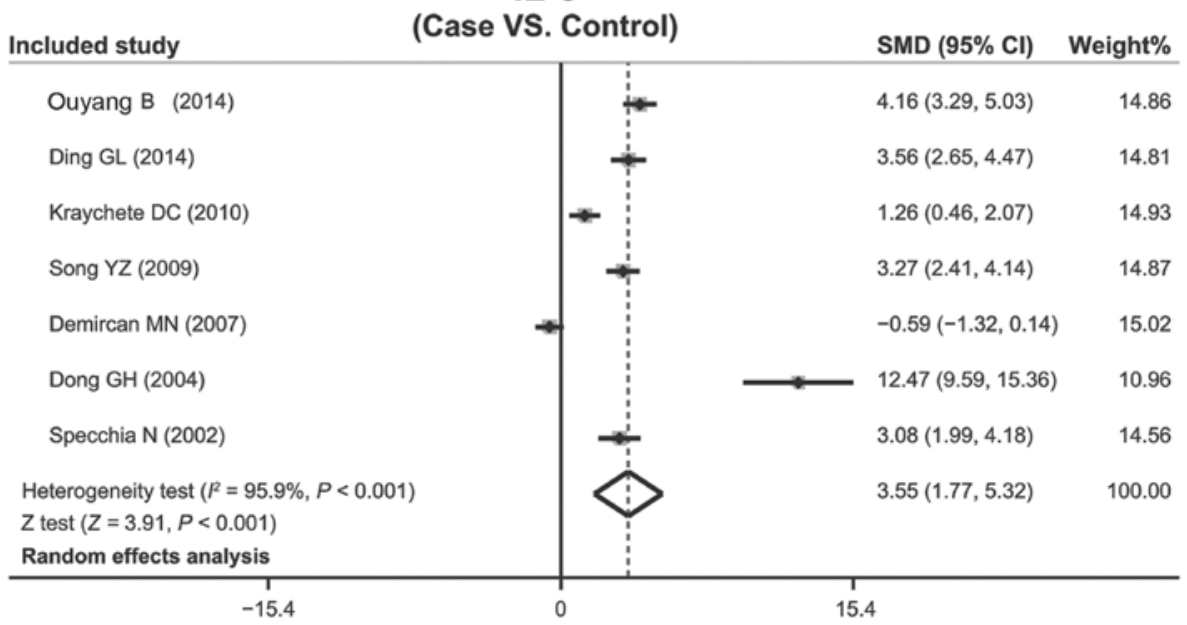

B

IL-6

\begin{tabular}{|c|c|c|c|}
\hline Included study & (Bulging VS. Control) & SMD $(95 \% \mathrm{Cl})$ & Weight $\%$ \\
\hline Ding GL (2014) & & $2.61(1.52,3.69)$ & 41.68 \\
\hline LiH (2010) & & $2.38(1.47,3.30)$ & 58.32 \\
\hline $\begin{array}{l}\text { Heterogeneity test }\left(R^{2}=0.00 \%, P=0.757\right) \\
Z \text { test }(Z=6.94, P<0.001)\end{array}$ & & $2.48(1.78,3.18)$ & 100.00 \\
\hline-3.69 & 0 & & \\
\hline
\end{tabular}

C

IL-6

Included study

(Protrusion VS. Control)

\begin{tabular}{|c|c|c|}
\hline Included study & SMD $(95 \%$ Cl) & Weight $\%$ \\
\hline Ding GL (2014) & $3.86(2.67,5.05)$ & 33.83 \\
\hline Li H (2010) & $3.06(2.21,3.91)$ & 66.17 \\
\hline $\begin{array}{l}\text { Heterogeneity test }(R=12.4 \%, P=0.285) \\
Z \text { test }(Z=9.45, P<0.001)\end{array}$ & $3.33(2.64,4.02)$ & 100.00 \\
\hline
\end{tabular}

D

IL-6

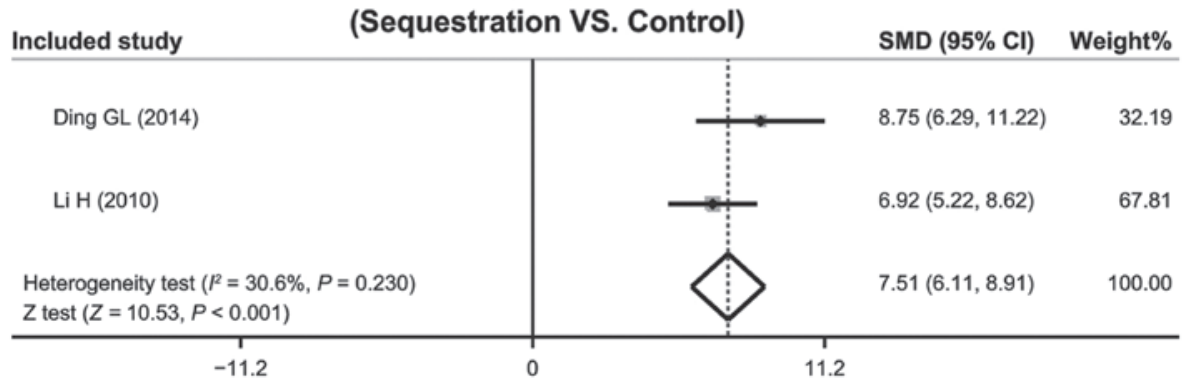

Figure 3. Forest plots depicting the correlation between the serum interleukin-6 (IL-6) protein expression levels and (A) intervertebral disc degeneration (IDD), and the (B) bulging; (C) protrusion; and (D) sequestration IDD subtypes. SMD, standardized mean difference; 95\% CI, 95\% confidence interval.

influence on the SMD comparisons between patients with IDD and healthy individuals (Fig. 4), and between patients with the various subtypes of IDD and healthy individuals
(Fig. 5). The funnel plots of IL-6 protein expression levels were symmetrical, and the Egger's linear regression test indicated no publication bias ( $>0.05)$ (Fig. 4). However, a 

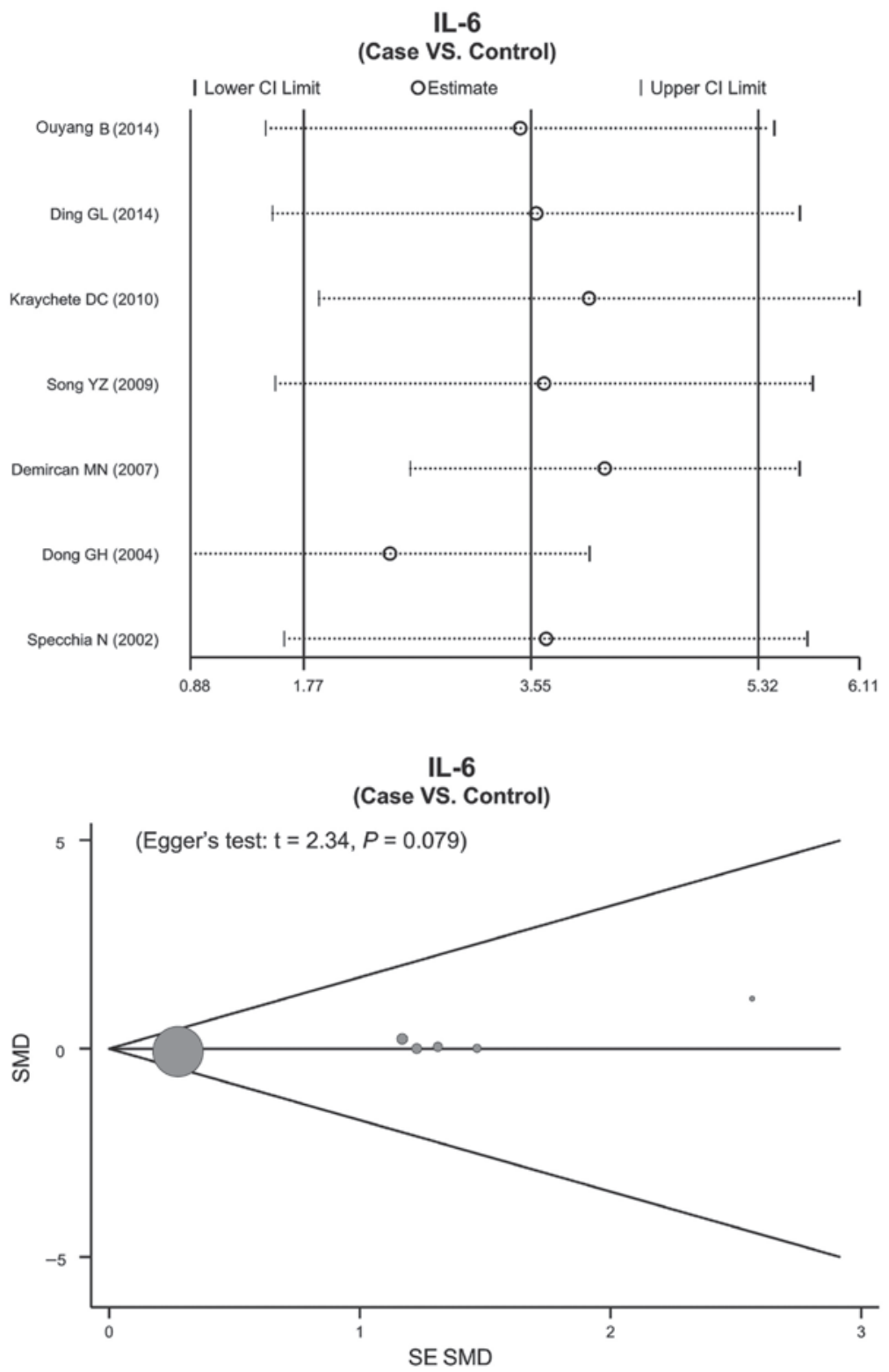

Figure 4. Sensitivity analyses of the correlations between the serum interleukin-6 (IL-6) protein expression levels and intervertebral disc degeneration.. CI, 95\% confidence interval; SMD, standardized mean difference.

specific P-value could not be determined, due to only two studies having reported on the expression levels of the IL-6 protein in the various IDD subtypes (Fig. 5).

Regression analysis. The results from the single factor meta-regression analysis suggested that the observed heterogeneity was independent of the year of submission, sample size, country and language (year of submission, $\mathrm{P}=0.589$; sample size, $\mathrm{P}=0.688$; country, $\mathrm{P}=0.300$; language, $\mathrm{P}=0.159$ ) (Fig. 6), and the results from multi-factor meta-regression analysis further demonstrated that ethnicity, year of submission, language and sample size did not contribute to the observed heterogeneity (Table II).

\section{Discussion}

IDD is a significant clinical and financial burden, due to the high rate of disability associated with the disease; pronounced physical defects occur in $10 \%$ of patients with IDD, stressing the importance of understanding the onset and progression of the disease (32). In the present study, a meta-analysis of published studies from numerous databases was conducted, in order to identify a definitive correlation between serum IL-6 expression levels, and IDD disease pathogenesis and severity. The protein expression levels of IL-6 were consistently higher in IVD protrusion tissue, as compared with normal IVD tissue. Furthermore, IL-6 protein expression levels were demonstrated to increase with increasing disease severity; thus suggesting that IL-6 may effect IDD disease progression. IVD predominantly consists of an inner nucleus pulposus (NP), surrounded by the annulus fibrosus and hyaline cartilaginous end-plate, which lie between adjacent vertebrae in the spine. The gelatinous NP is regarded as an avascular tissue containing ECM, which consists of hydrated proteoglycan and collagen (33). IDD has previously been demonstrated to be associated with degradation of the ECM in the NP, and 
Table II. Meta-regression analyses of potential sources of heterogeneity.

\begin{tabular}{|c|c|c|c|c|c|c|}
\hline \multirow[b]{2}{*}{ Heterogeneity factors } & \multirow[b]{2}{*}{ Coefficient } & \multirow[b]{2}{*}{$\mathrm{SE}$} & \multirow[b]{2}{*}{$\mathrm{t}$} & \multirow[b]{2}{*}{ P-value (adjusted) } & \multicolumn{2}{|c|}{$95 \% \mathrm{CI}$} \\
\hline & & & & & LL & UL \\
\hline Year & -0.876 & 0.564 & -1.55 & 0.476 & -3.301 & 1.55 \\
\hline Sample size & 0.059 & 0.209 & 0.28 & 0.939 & -0.841 & 0.958 \\
\hline Country & -2.712 & 3.37 & -0.8 & 0.703 & -17.214 & 11.789 \\
\hline Language & -1.451 & 8.204 & -0.18 & 0.99 & -36.75 & 33.848 \\
\hline
\end{tabular}

SE, standard error; 95\% CI, 95\% confidence interval, LL, lower limit; UL, upper limit.

A

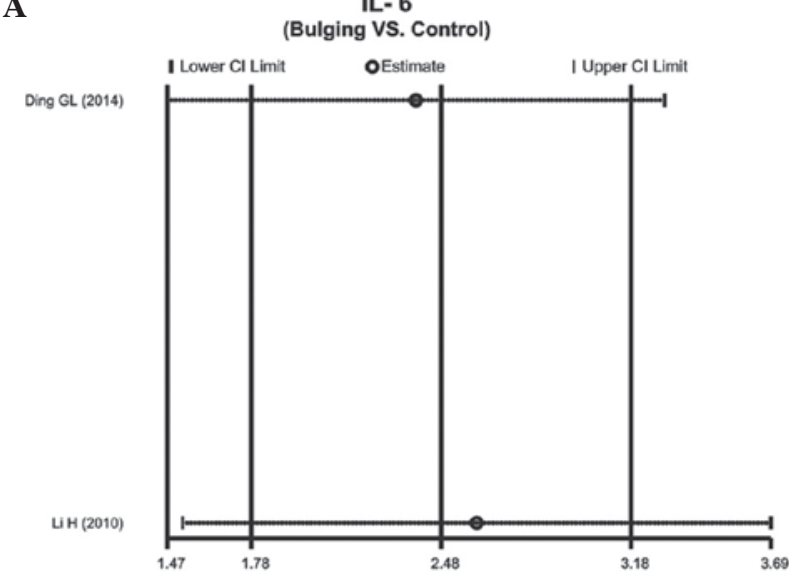

B

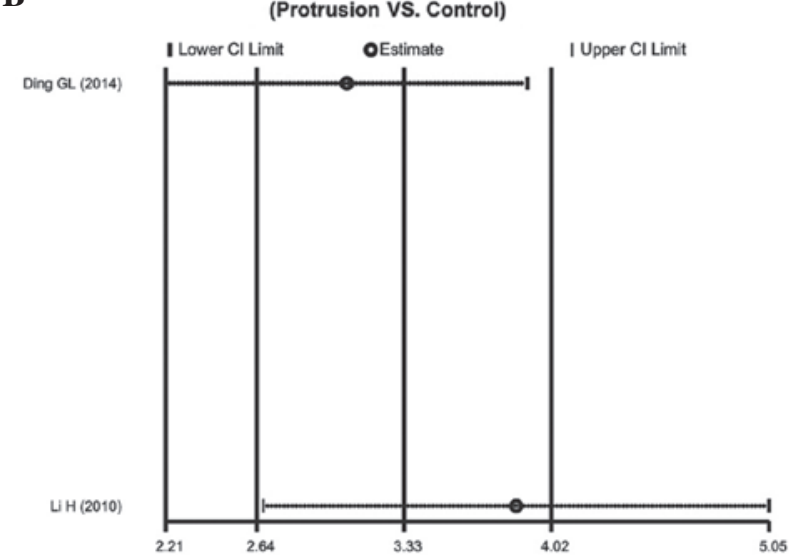

C

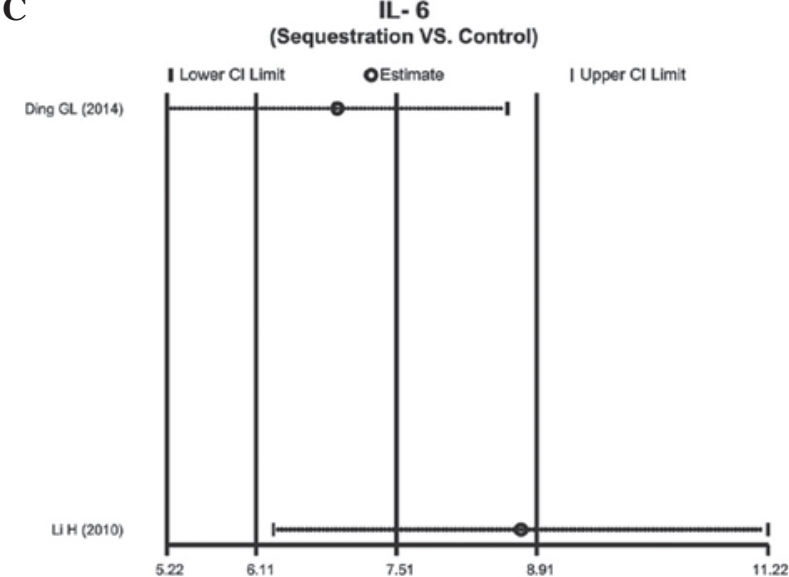

IL- 6

(Bulging VS. Control)

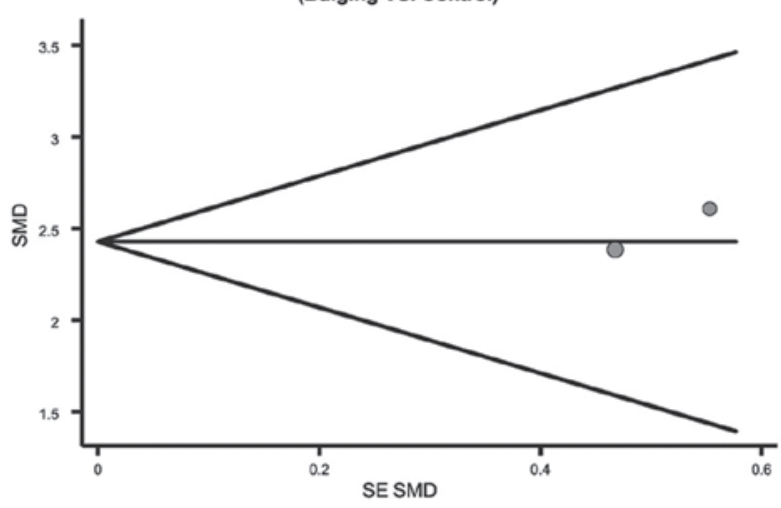

IL- 6

(Protrusion VS. Control)

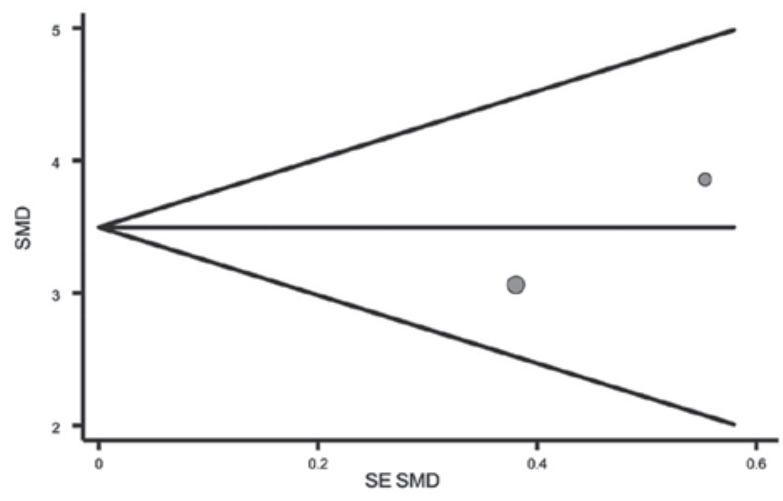

IL- 6

(Sequestration VS. Control)

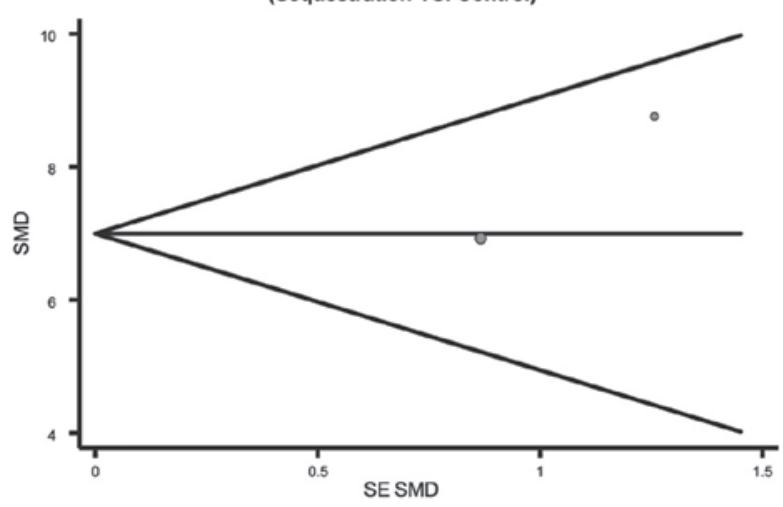

Figure 5. Sensitivity analyses of the correlations between the serum interleukin-6 (IL-6) protein expression levels and the various intervertebral disc degeneration (IDD) subtypes: (A) Bulging, (B) protrusion and (C) sequestered IDD subtypes. 
A

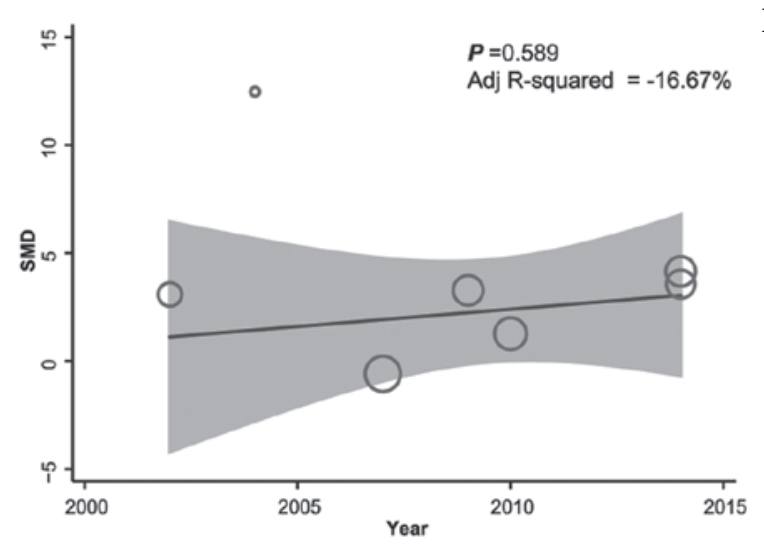

C

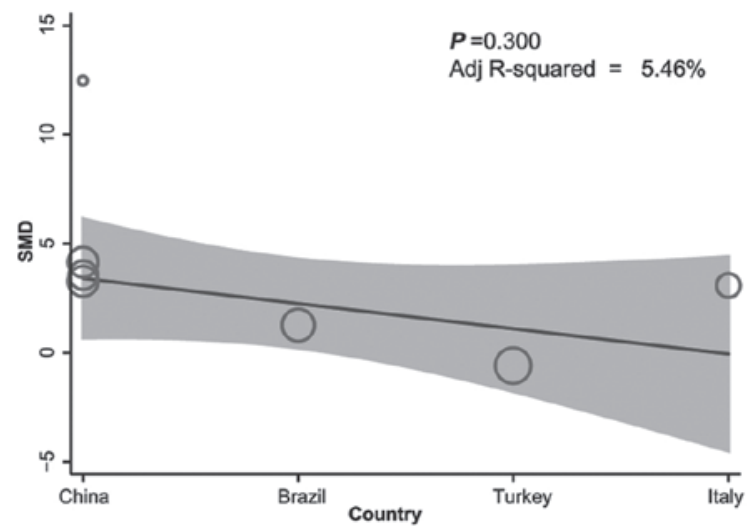

B
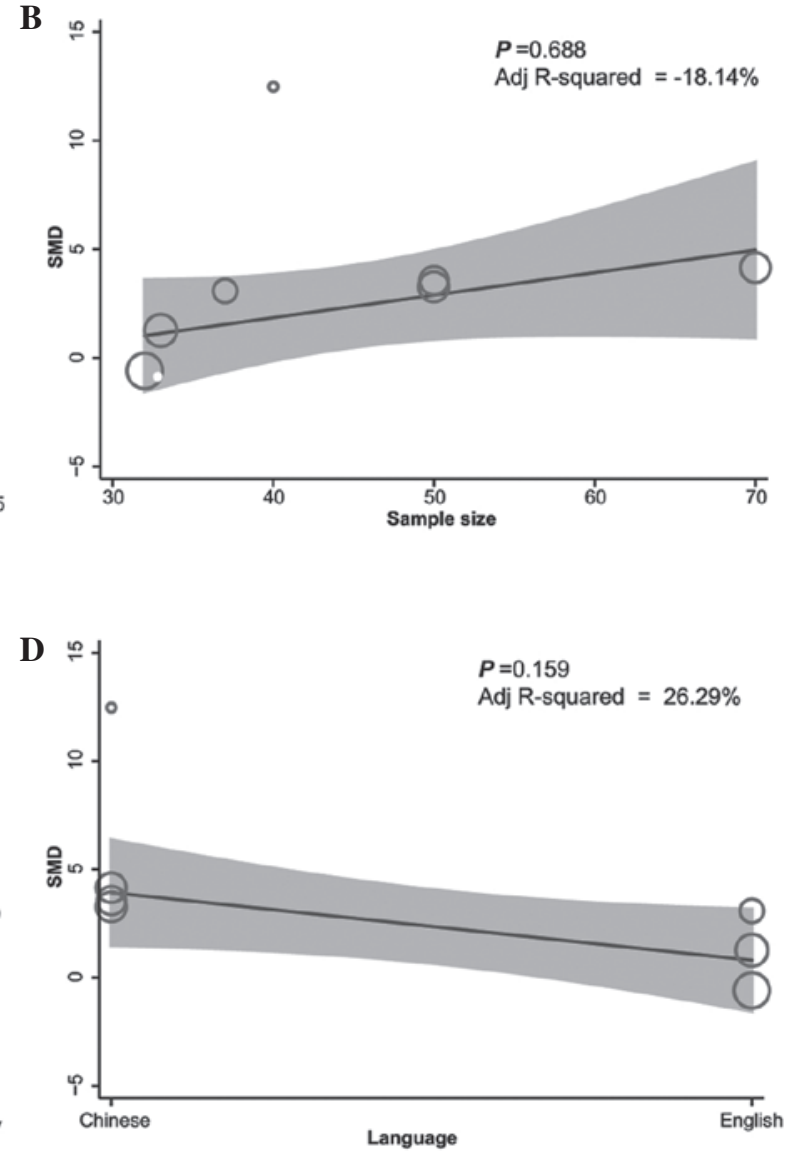

Figure 6. Meta-regression analyses of the correlations between the serum interleukin-6 protein expression levels and the various intervertebral disc degeneration subtypes. The results suggested that the heterogeneity was independent of (A) the year of submission, (B) the sample size, (C) the country of submission, and (D) the language of the study. SMD, standardized mean difference.

decreased levels of disc ECM proteoglycans (2). Furthermore, IDD has been associated with alterations in the collagen type content of the ECM, decreased water disc content, and local inflammatory reactions (34). In a previous analysis of human articular cartilage, IL-6 inhibited proteoglycan synthesis, which maintains high NP tissue hydration, and prevents blood and lymphatic vessel ingrowth, under normal physiological conditions (19). Degrading IVD tissue has been demonstrated to secrete IL-6 and other inflammatory cytokines (35); therefore, this may explain the upregulated expression levels of IL-6 in IVD protrusion tissue, as compared with normal IVD tissue.

In order to assess factors confounding the validity of the results, the present meta-analysis investigated whether IL-6 protein expression levels varied between the different IDD clinical subtypes (bulging, protrusion and sequestered), as compared with the controls. The serum IL- 6 expression levels were similarly upregulated in IVD protrusion tissue, as compared with normal IVD tissue, irrespective of the IDD subtype, and the IL-6 levels increased with increasing severity of IDD.

The limitations of the present meta-analysis include its similarity to other published meta-analyses and the small sample size; of the 112 studies initially identified, only eight met the inclusion criteria and this may have negatively impacted on the reliability of the results. In addition, a subgroup analysis based on ethnicity could not be performed, which may have indicated ethnicity bias in the results.

In conclusion, the present meta-analysis demonstrated that the protein expression levels of IL- 6 were upregulated in IVD protrusion tissue, as compared with normal IVD tissue, and this was irrespective of the IDD subtype. Therefore, IL-6 expression levels may effect the progression of IDD. Future studies should endeavor to elucidate the underlying mechanism by which IL-6 effects the onset and progression of IDD.

\section{References}

1. Wang HQ, Yu XD, Liu ZH, Cheng X, Samartzis D, Jia LT, Wu SX, Huang J, Chen J and Luo ZJ: Deregulated miR-155 promotes Fas-mediated apoptosis in human intervertebral disc degeneration by targeting FADD and caspase-3. J Pathol 225: 232-242, 2011.

2. Lee JM, Song JY, Baek M, Jung HY, Kang H, Han IB, Kwon YD and Shin DE: Interleukin-1 $\beta$ induces angiogenesis and innervation in human intervertebral disc degeneration. J Orthop Res 29: 265-269, 2011.

3. Siemionow K, An H, Masuda K, Andersson G and Cs-Szabo G: The effects of age, sex, ethnicity, and spinal level on the rate of intervertebral disc degeneration: A review of 1712 intervertebral discs. Spine (Phila Pa) 36: 1333-1339, 2011.

4. Akhatib B, Onnerfjord P, Gawri R, Ouellet J, Jarzem P, Heinegård D, Mort J, Roughley P and Haglund L: Chondroadherin fragmentation mediated by the protease HTRA1 distinguishes human intervertebral disc degeneration from normal aging. J Biol Chem 288: 19280-19287, 2013. 
5. Phillips KL, Jordan-Mahy N, Nicklin MJ and Le Maitre CL Interleukin-1 receptor antagonist deficient mice provide insights into pathogenesis of human intervertebral disc degeneration. Ann Rheum Dis 72: 1860-1867, 2013.

6. Hughes SP, Freemont AJ, Hukins DW, McGregor AH and Roberts S: The pathogenesis of degeneration of the intervertebral disc and emerging therapies in the management of back pain J Bone Joint Surg Br 94: 1298-1304, 2012.

7. Adams MA and Dolan P: Intervertebral disc degeneration: Evidence for two distinct phenotypes. J Anat 221: 497-506, 2012.

8. Huang M, Wang HQ, Zhang Q, Yan XD, Hao M and Luo ZJ: Alterations of ADAMTSs and TIMP-3 in human nucleus pulposus cells subjected to compressive load: Implications in the pathogenesis of human intervertebral disc degeneration. J Orthop Res 30: 267-273, 2012.

9. Erwin WM, Islam D, Inman RD, Fehlings MG and Tsui FW: Notochordal cells protect nucleus pulposus cells from degradation and apoptosis: Implications for the mechanisms of intervertebral disc degeneration. Arthritis Res Ther 13: R215, 2011.

10. Mwale F, Masuda K, Pichika R, Epure LM, Yoshikawa T, Hemmad A, Roughley PJ and Antoniou J: The efficacy of Link N as a mediator of repair in a rabbit model of intervertebral disc degeneration. Arthritis Res Ther 13: R120, 2011.

11. Miyagi M, Ishikawa T, Orita S, Eguchi Y, Kamoda H, Arai G Suzuki M, Inoue G, Aoki Y, Toyone T, et al: Disk injury in rats produces persistent increases in pain-related neuropeptides in dorsal root ganglia and spinal cord glia but only transient increases in inflammatory mediators: Pathomechanism of chronic diskogenic low back pain. Spine (Phila Pa) 36: 2260-2266, 2011.

12. Hirano T, Yasukawa K, Harada H, Taga T, Watanabe Y, Matsuda T, Kashiwamura S, Nakajima K, Koyama K, Iwamatsu A et al: Complementary DNA for a novel human interleukin (BSF-2) that induces B lymphocytes to produce immunoglobulin. Nature 324: 73-76, 1986.

13. Neurath MF and Finotto S: IL-6 signaling in autoimmunity, chronic inflammation and inflammation-associated cancer. Cytokine Growth Factor Rev 22: 83-89, 2011.

14. Ataie-Kachoie P, Pourgholami MH and Morris DL: Inhibition of the IL-6 signaling pathway: A strategy to combat chronic inflammatory diseases and cancer. Cytokine Growth Factor Rev 24: 163-173, 2013.

15. Yao J, Feng XW, Yu XB, Xie HY, Zhu LX, Yang Z, Wei BJ, Zheng SS and Zhou L: Recipient IL-6-572C/G genotype is associated with reduced incidence of acute rejection following liver transplantation. J Int Med Res 41: 356-364, 2013.

16. Heikkilä K, Ebrahim S and Lawlor DA: Systematic review of the association between circulating interleukin-6 (IL-6) and cancer. Eur J Cancer 44: 937-945, 2008

17. Studer RK, Vo N, Sowa G, Ondeck C and Kang J: Human nucleus pulposus cells react to IL-6: Independent actions and amplification of response to IL-1 and TNF- $\alpha$. Spine (Phila Pa 1976) 36 593-599, 2011.

18. Huang KY, Lin RM, Chen WY, Lee CL, Yan JJ and Chang MS IL-20 may contribute to the pathogenesis of human intervertebral disc herniation. Spine (Phila Pa 1976) 33: 2034-2040, 2008.

19. Hamamoto H, Miyamoto H, Doita M, Takada T, Nishida K and Kurosaka M: Capability of nondegenerated and degenerated discs in producing inflammatory agents with or without macrophage interaction. Spine (Phila Pa 1976) 37: 161-167, 2012.
20. Liu Z, Tang NL, Cao XB, Liu WJ, Qiu XS, Cheng JC and Qiu Y: Lack of association between the promoter polymorphisms of MMP-3 and IL-6 genes and adolescent idiopathic scoliosis: A case-control study in a Chinese Han population. Spine (Phila Pa 1976) 35: 1701-1705, 2010.

21. Jackson D, White IR and Riley RD: Quantifying the impact of between-study heterogeneity in multivariate meta-analyses. Stat Med 31: 3805-3820, 2012.

22. Peters JL, Sutton AJ, Jones DR, Abrams KR and Rushton L: Comparison of two methods to detect publication bias in meta-analysis. JAMA 295: 676-680, 2006.

23. Zintzaras E and Ioannidis JP: Heterogeneity testing in meta-analysis of genome searches. Genet Epidemiol 28: 123-137, 2005.

24. Ouyang B, Su JC, Zeng YD and Bao J: Expression and significance of IL-6, IL-10 and MCP-1 in degenerate human lumbar intervertebral disc tissues. Hainan Yixueyuan Xuebao 3: 381-383, 2014 (In Chinese).

25. Ding GL, Li XY and Lv MX: Expression and clinical study of IL-1 and IL-6 in lumbar disc herniation. Chongqing Yixue 43: 1919-1922, 2014 (In Chinese).

26. Li H: Expression and significance of protrusion of intervertebral disc degeneration in patients with lumbar disc tissue in IL-6, IL-8, TNF-alpha. Shangdong Yixue 50: 65-66, 2010 (In Chinese).

27. Kraychete DC, Sakata RK, Issy AM, Bacellar O, Santos-Jesus R and Carvalho EM: Serum cytokine levels in patients with chronic low back pain due to herniated disc: Analytical cross-sectional study. Sao Paulo Med J 128: 259-262, 2010.

28. Song YZ and Deng XZ: Detection and significance of lumbar intervertebral disc nucleus pulposus tissue TNF-alpha, IL-1, IL-6. Shangdong Yixue 49: 87-88, 2009 (In Chinese).

29. Demircan MN, Asir A, Cetinkal A, Gedik N, Kutlay AM, Colak A, Kurtar S and Simsek H: Is there any relationship between proinflammatory mediator levels in disc material and myelopathy with cervical disc herniation and spondylosis? A non-randomized, prospective clinical study. Eur Spine J 16: 983-986, 2007.

30. Dong GH, Huang JQ, Cai XJ, Han JH and Xia BJ: Expression of IL-1 and IL-6 in the herniated lumbar disc tissues. Zhongguo Yishi Zazhi 6: 1134-1135, 2004 (In Chinese).

31. Specchia N, Pagnotta A, Toesca A and Greco F: Cytokines and growth factors in the protruded intervertebral disc of the lumbar spine. Eur Spine J 11: 145-151, 2002.

32. Wilke HJ, Urban J and Kümin M: The benefits of multi-disciplinary research on intervertebral disc degeneration. Eur Spine J 23 (Suppl 3): S303-S304, 2014.

33. Sakai D, Nakamura Y, Nakai T, Mishima T, Kato S, Grad S, Alini M, Risbud MV, Chan D, Cheah KS et al: Exhaustion of nucleus pulposus progenitor cells with ageing and degeneration of the intervertebral disc. Nat Commun 3: 1264, 2012.

34. Kalichman L and Hunter DJ: The genetics of intervertebral disc degeneration. Associated genes. Joint Bone Spine 75: 388-396, 2008.

35. Holm S, Mackiewicz Z, Holm AK, Konttinen YT, Kouri VP, Indahl $\mathrm{A}$ and Salo J: Pro-inflammatory, pleiotropic, and anti-inflammatory TNF-alpha, IL-6, and IL-10 in experimental porcine intervertebral disk degeneration. Vet Pathol 46: 1292-1300, 2009. 